\title{
El nuevo localismo democrático: análisis descriptivo de algunas tendencias *
}

\section{Clemente J. Navarro Yáñez **}

Desde el nacimiento de la democracia se ha postulado la necesidad de establecer comunidades de tamaño reducido para garantizar la participación activa de los ciudadanos en las labores de gobiemo. En la actualidad este postulado se sigue manteniendo, pero se añade otro conjunto de premisas referidas a las ventajas que posee el gobiemo municipal con respecto a otros de mayor escala por lo que se refiere a la eficacia de la acción gubernamental.

En ello han contado también las propias iniciativas de los actores locales, algunas de las cuales han sido recogidas posteriormente en la legislación de ámbito nacional, así como en el diseño institucional que el Estado central ha realizado con respecto a los gobiemos municipales.

Denominaremos nuevo localismo democrático a este conjunto de iniciativas y reflexiones que subrayan las posibilidades del ámbito municipal para poner en marcha procesos y reformas institucionales con el fin de democratizar la labor de gobierno.

El primer apartado se dedica a definir de una forma más extensa este fenómeno. En el segundo, núcleo central del artículo, se presenta una descripción básica de algunas de las tendencias y estrategias por las que viene desarrollándose. Este análisis se basa en un estudio exploratorio de experiencias desarrolladas en municipios de diversas democracias occidentales.

\section{Escala y gobierno democrático: el nuevo localismo democrático}

Existe un consenso bastante generalizado acerca de que a medida que la escala es más reducida, mayores son las posibili- dades de que los ciudadanos participen directamente en las labores de gobierno (DAHL y TUFTE, 1973).

Por ello, la reflexión de la teoría democrática acerca de las insuficiencias de oportunidades de participación que ofrece la democracia representativa a nivel nacional, ha visto en la escala municipal el locus en el que desarrollar procesos de carácter democratizador.

En general, la literatura que desde los años sesenta aborda la cuestión de la democracia participativa, señala al gobierno municipal, o incluso a escalas más reducidas, como el lugar privilegiado para la puesta en marcha de reformas institucionales que, sin socavar el principio representativo legitimado mediante la competencia electoral, den lugar a una mayor implicación de los ciudadanos en las labores de gobierno, ya sea directamente, o mediante grupos de representación de intereses ${ }^{1}$.

A diferencia de las posturas clásicas sobre el tema, que insistían únicamente en la dimensión participativa, la ciencia politica señala la posibilidad de nuevas contribuciones del ámbito municipal a la dimensión política de las sociedades contemporáneas. A saber: las referidas al aumento en la eficacia en la provisión de servicios. Esta apreciación es pertinente en un momento como el actual, en el que los municipios constituyen una de las vías principales por las que se canaliza la provisión de servicios de bienestar, desde los de urbanización a los servicios de atención a grupos específicos? 2 .

Así, por ejemplo, se postula que la cercanía existente entre oferta (gobierno municipal) y demanda (ciudadanos) facilita una mejor y más flexible adecuación entre ellas, puede ayudar a reducir los costes informativos que toda política pública conlleva, o aumenta las posibilidades de coordinación en comparación con la acción que tiene lugar mediante agencias gubemamentales de carácter sectorial (CLARKE y GAILE, 1989). 
Estas consideraciones no pertenecen únicamente al ámbito de la teoría política. También en la práctica, los actores locales vienen poniendo en marcha procesos concretos para desarrollar estas potencialidades. En este sentido vienen surgiendo iniciativas locales para favorecer la participación de los ciudadanos en la política municipal o, por ejemplo, para aumentar su capacidad de elección entre diversos servicios.

Denominaremos aquí nuevo localismo democrático a esta tendencia conjunta, en la que convergen teoría y práctica, que añade a las aportaciones clásicas y recientes sobre participación un nuevo valor: el aumento de la eficacia de la acción gubernamental. En el siguiente apartado se describe este fenómeno.

\section{Tendencias y estrategias en el nuevo localismo democrático}

El primer acercamiento a las experiencias de nuevo localismo democrático revela que existen tantos modelos como casos. En un análisis micro, en el que se atendiera a cada gobiemo local en particular, podría argumentarse que el nuevo localismo democrático presenta tantas formas como municipios existen. Sin embargo, es posible centrarse en un nivel de análisis más amplio que identifique modelos y tendencias, en los que quede reflejada la diversidad que se encuentra en los casos particulares.

Desde esta perspectiva, en el conjunto de experiencias analizadas pueden distinguirse dos tendencias básicas, que se articulan en función de las ventajas relativas - potencialidades que ofrece el gobierno local con respecto a otros de mayor escala: la eficacia en la provisión de servicios y el desarrollo de procesos de democratización. Dentro de cada una de estas tendencias pueden describirse, a su vez, diferentes estrategias, vías o mecanismos concretos por los que los actores implicados pretenden alcanzar los objetivos que guían su acción.

Antes de entrar en la descripción de cada una de ellas conviene advertir que la taxonomía que se presenta no pretende ser exhaustiva, por cuanto es reflejo de un estudio de carácter exploratorio. Con éste se pretende únicamente sentar las bases para realizar posteriormente un análisis comparado más amplio. Por otra parte, la distinción establecida responde fundamentalmente a criterios de orden analítico, de tal forma que en un mismo municipio pueden darse diversas estrategias, al mismo tiempo que mediante una misma estrategia se pretende alcanzar objetivos diversos. Aún así, ha parecido pertinente presentar las experiencias agrupadas en tendencias que, en su conjunto, dan cuenta de grupos homogéneos ${ }^{3}$.

\subsection{Eficacia y provisión de servicios: consumidores, capacidad de elección y "estados permisivos”}

Una de las tendencias identificable agrupa aquellas experiencias que han hecho del aumento de la eficacia y la competitividad sus objetivos principales. En estas experiencias se trata tanto de afrontar los problemas derivados de la crisis de las haciendas locales, como de ampliar la capacidad de elección de los ciudadanos, de manera que haya un mayor grado de adecuación entre las preferencias individuales y los servicios que provee el gobierno municipal.

Durante la década de los sesenta y los setenta, la provisión de servicios se había caracterizado por ser de corte •burocrático-paternalista. (BLACKMAN, 1995). El rasgo más sobresaliente en su dimensión organizativa era el establecimiento de modelos burocráticos. Los servicios se destinan a grupos delimitados por estar afectados por determinados problemas sociales, a través de análisis realizados por especialistas. De esta forma, tanto su provisión, como sus características, tendían a depender más de los análisis realizados y de la interpretación que de éstos hacían los especialistas, que de las preferencias individuales.

A mediados de los años 70 , y de forma más nítida a partir de la década de los 80 , se plantea la necesidad de cambiar este modelo. Ello se debe principalmente a tres factores. En primer lugar, se constata el incremento constante del gasto público, que ha producido la crisis fiscal del Estado, y que incide de una forma particular en los gobiernos locales ${ }^{4}$. A ello se une la ruptura del consenso anteriormente existente entre todos los partidos políticos acerca de la necesidad de aumentar tanto el volumen como la cobertura de los servicios de bienestar. Por último, el creciente escepticismo entre políticos y ciudadanos acerca de la adecuación entre servicios y necesidades o preferencias individuales 5 .

Las soluciones desarrolladas ante estas cuestiones han sido, por un lado, la incorporación de métodos propios del sector privado en la provisión de servicios públicos, con la intención de potenciar la adecuación entre servicios (oferta) y preferencias individuales (demanda) y, por otro, la apertura a otros agentes provisores de bienestar, además del Estado.

Frente al modelo de gestión aburocrático-paternalista tiende a desarrollarse un modelo "post-burocrático. (BLACKMAN, 1995) Su principal objetivo es la modernización de las administraciones municipales. Este modelo se caracteriza por una tendencia a la desregulación, la fexibilización y la atención a los individuos como consumidores de servicios. Para ello se desarrollan métodos de gestión propios del sector privado, mediante la fija- 
ción de estándares y objetivos por parte de las agencias gubernamentales y los individuos interesados. A ello se une el uso de nuevas tecnologías con el fin de aumentar la eficacia en la comunicación y favorecer el acceso a la información por parte de los individuos. En esencia, se trata de adecuar las estructuras organizativas existentes a modelos de trabajo y gestión propios del sector privado, bajo el supuesto de que, al igual que en el mercado, se producirán autorregulación y adecuación entre servicios y necesidades, aunque el proceso de provisión de servicios continúa siendo de carácter público.

Esta estrategia ha sido desarrollada casi en la totalidad de los municipios europeos, aunque en menor medida en la Europa Meridional. Ejemplo de ello es la estrategia de modernización desarrollada en la administración pública municipal en Alemania Occidental ${ }^{6}$ o las iniciativas desarrolladas a través del programa •Free Local Government en los Países Nórdicos ${ }^{7}$.

Otra modalidad, propia del Reino Unido, ha sido la redacción de aCartas Ciudadanas. (Citizens' Charters) desde inicios de los noventa, como por ejemplo la City Council's Citizen's Charter de la ciudad de York (1989). Otro ejemplo posterior es el de la County Council Citizen's Charter de Hertforshire (1993). En ellas se trata de explicitar los aderechos de los consumidores de servicios públicos, entre los que se encuentra su participación en los procesos de planificación y control, para con ello aumentar la calidad y el grado de adecuación de los servicios a las necesidades de los consumidores. Se fijan objetivos, estándares, indicadores, financiación y procesos a desarrollar en la provisión de un determinado servicio ${ }^{8}$. A diferencia de la estrategia señalada anteriormente, se abre la posibilidad de que otros agentes, además del Estado, se conviertan en proveedores ${ }^{9}$.

La apertura a otros agentes provisores ha hecho de los gobiernos municipales "estados permisivos" (enabling states), agentes que promueven el bienestar de los ciudadanos tanto de forma directa como a través de organizaciones no-gubernamentales. En general, esta estrategia parece ser una tendencia común de los gobiernos locales de la totalidad de las democracias occidentales ${ }^{10}$. Suele desarrollarse de tres formas: privatización, contratos con agencias no gubernamentales de carácter lucrativo y a través de organizaciones voluntarias. En la primera, los gobiemos locales dejan todo el proceso de producción de servicios a cargo de entidades privadas. En las otras, el Estado sigue controlando este proceso, aunque la provisión directa a los ciudadanos la realicen organizaciones no gubernamentales.

La privatización de los servicios municipales ligados a la planificación y urbanización (agua, gas, electricidad, vivien$\mathrm{da}, . .$.$) viene siendo una práctica común en la totalidad de los$ gobiernos municipales. O, cuando menos, lo ha sido la crea-

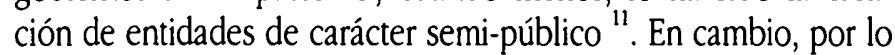
que respecta a los servicios de carácter personal, o más en ge- neral, a los servicios sociales (infancia, tercera edad, bienestar social, cultura, deportes,...), la tendencia ha consistido en la diversificación y la complementación entre sectores público y privado, más que en la privatización ${ }^{12}$. En Norteamérica y Gran Bretaña coexisten dinámicas privatizadoras con la inserción de organizaciones no lucrativas ${ }^{13}$. En cambio, en Europa Central y los Países Nórdicos, la estrategia dominante viene siendo la provisión a través de entidades privadas, más que la generación de situaciones de mercado, $y$, sobre todo, a través de convenios con organizaciones voluntarias ${ }^{14}$. Esta última ha sido la estrategia básica en la Europa Meridional, aunque en menor medida que en los otros países ${ }^{15}$.

Así pues, a nivel macro puede indicarse que la provisión de servicios viene desarrollándose mediante procesos que centran su atención sobre el aumento en las posibilidades de elección de los ciudadanos como consumidores de servicios. Esto se ha intentado lograr mediante la modernización de la administración pública o a través de la diversificación de la oferta. Para esto último se ha recurrido a la privatización o al desarrollo de convenios con entidades privadas, tanto de carácter lucrativo como no lucrativo. A nivel meso, se constata que el grado en que las estrategias señaladas están presentes en los países analizados no es similar. La modernización de la administración pública ha sido diferente en el norte y el sur de Europa. La privatización se da en mayor medida en Gran Bretaña y Norteamérica. El convenio con entidades privadas aparece ligado a la Europa Central y los Países Nórdicos, y en menor medida, a la Europa Meridional.

\subsection{Participación ciudadana: democratización y oportunidades de participación política}

En la tendencia que se describe a continuación, el objeto fundamental ha sido el desarrollo de iniciativas tendentes a promocionar y favorecer la participación de los ciudadanos en los procesos de toma de decisiones referidos a la política municipal. Han tenido como objeto favorecer la inclusión de los ciudadanos en los procesos de toma de decisiones, ofreciendo oportunidades de participación. El desarrollo de este modelo ha seguido principalmente dos estrategias. El establecimiento de procedimientos o canales concretos de participación, o bien el desarrollo e institucionalización de un sistema general de regulación de la participación ciudadana, en el que suelen incluirse los procedimientos anteriores.

Entre éstos destacan tres: referéndums y consultas, consejos $u$ órganos consultivos y procesos de descentralización. Los referéndums son una de las formas típicas utilizadas en Norteamérica, y principalmente en los Estados Unidos (CHANDLER, 1993). El uso del referéndum es menos común en Europa. Aún así, ha 
existido alguna èxperiencia, especialmente en los Países Nórdicos, Suiza y recientemente en Italia (GuSTAFFSON, 1991). A ello se une que, por lo general, y a diferencia de los norteamericanos, los municipios en Europa poseen escasa autonomía para convocarlos. Aunque suelen realizarse procesos consultivos de carácter no vinculante mediante encuestas o estudios de opinión ${ }^{16}$.

El procedimiento más extendido ha sido la constitución de órganos de carácter consultivo. Suelen tomar la forma de comités, con funciones de asesoramiento y consulta respecto a los consejos municipales. Se conforman territorialmente por barrios o distritos, o bien funcionalmente, en correspondencia con las competencias de los consejos o agencias gubernamentales existentes. Una de sus características es la inclusión de aquellas organizaciones ciudadanas que abordan el área de trabajo o política que se trate.

En Canadá, por ejemplo, se crearon a finales de los años setenta "grupos de asesoramiento de residentes" (residents' advisory groups) durante la reforma del área metropolitana de Winnipeg (Winnipeg Unicity). Estos grupos estaban formados por miembros de organizaciones ciudadanas elegidos por los residentes. Su labor consistía en asesorar a los órganos municipales o comités territoriales mediante la organización de reuniones abiertas a todos los residentes.

En Europa es común la constitución de consejos consultivos en relación con las áreas de trabajo de los gobiernos municipales. En Francia, por ejemplo, se han generalizado la formación de "grupos de trabajo (atelier du travail) para abordar problemas de urbanismo en ciudades o zonas concretas (barrios). En el marco del programa "Free Local Govermment, se han creado consejos consultivos en cada uno de los consejos municipales de los municipios noruegos ${ }^{17}$.

Los procesos de descentralización son comunes en toda la Europa Continental ${ }^{18}$. Su objetivo ha sido acercar los procesos de toma de decisiones a los ciudadanos, sobre todo, en los municipios de gran tamaño. Durante los años setenta tuvo lugar en los países de la Europa Central y la región nórdica un proceso de agregación de las entidades locales para lograr una mayor eficiencia en la provisión de servicios (HiLl, 1980). Según Gru. NOw (1991). Ello produjo un incremento en la distancia espacial y comunicativa existente entre el gobierno local y los ciudadanos y, por tanto, una reducción en las posibilidades que los últimos tenían para participar. Algunos gobiernos locales han creado órganos de carácter sub-municipal para intentar remediar este problema. Éste es el caso, por ejemplo, de Alemania Occidental.

Estos procesos de descentralización también han sido desarrollados en algunos de los municipios nórdicos y, sobre todo, entre los acogidos al programa "Free Local Government. En ciertos casos, como por ejemplo en algunos municipios suecos, además de funciones de asesoramiento, les han sido concedidas competencias generales sobre el área de trabajo que abordan $e$ incluso en otros se han llegado a suprimir los comités u órganos de gobierno municipales. Algunos ejemplos son las ciudades de Öbero, Eskilstuna o Umea ${ }^{19}$.

Otra de las formas en que se ha desarrollado esta tendencia ha sido la de los "Consejos de Circunscripción. (Consigli di Circonscripzione) en Italia. Con tales consejos se pretende tanto la descentralización de algunos servicios como la incorporación de los ciudadanos en los procesos de toma de decisiones. Así, por ejemplo, con la Ley n. 278 de 1976 y, posteriormente, en 1990 con la Ley sobre el Ordenamiento de la Autonomía Local, se les concede capacidad presupuestaria bajo la coordinación del consejo municipal (Consiglio Comunale) y se aumentan sus competencias (cultura, educación, servicios sociales, salud o deportes). En relación con esto último, se han incorporado organizaciones ciudadanas en los consejos y en los procesos de toma de decisiones que son de su competencia, principalmente en aquellas referidas a los servicios sociales ${ }^{20}$.

En el caso español también ha sido generalizada la creación de Consejos de Distritos (órganos de descentralización). Así, por ejemplo, alrededor del $70 \%$ de los municipios de 20.000 o más habitantes poseían este tipo de órganos a principios de los noventa (NAVARRO, 1994, 1997: 531).

La otra estrategia, desarrollada en municipios europeos, ha consistido en la creación de marcos de carácter jurídico-normativo en los que se recogen diversos instrumentos de participación, incluidos los comentados más arriba. Según estos textos, su principal objetivo es procurar la regulación global de los procesos de participación ciudadana. Esta estrategia ha tenido lugar, sobre todo, en la Europa Meridional y, concretamente, en España e Italia ${ }^{21}$. En estos dos países la legislación referida a los municipios, además de señalar aquellos instrumentos de participación que deben existir obligatoriamente en ellos, los facultan para desarrollar autónomamente todos aquellos instrumentos que puedan favorecer y fomentar la participación de los ciudadanos en el gobiemo local ${ }^{22}$. En el caso de Italia tanto la Norma sul Decentramento e sulla Partecipazione dei Cittadini nell'amministrazione del Comune (Ley n. 278 de 1976), como la reforma del régimen local realizada a partir del año 1990, recoge este asunto, concretamente en el capítulo III de la Ley sobre el "Ordinamento delle autonomie locale. En éste se especifican las fórmulas por las que los municipios deben propiciar la participación de los ciudadanos, como, por ejemplo, la elección de los miembros de la corporación municipal, la consulta, el referéndum, acción popular, el defensor cívico o la descentralización. Además, los municipios pueden diseñar su propio ‘stituti di Partecipazione, en el que pueden incluirse estos instrumentos y aquellos otros que crea pertinente el gobiemo municipal. 
En España la situación es similar. Además de los instrumentos recogidos en la Ley de Bases del Régimen Local y en la Ley sobre el Régimen de Organización y Funcionamiento de las Entidades Locales (intervención en Pleno Municipal, en Comisiones Informativas, iniciativa ciudadana, consulta popular, audiencia pública, consejos sectoriales y de distrito), los municipios han desamollado de forma autónoma Reglamentos de Participación Ciudadana. Éstos establecen un marco jurídico-normativo que delimita las relaciones que pueden establecer gobiernos locales y ciudadanía en cada municipio. Actualmente, gran parte de los municipios de 20.000 o más habitantes poseen Reglamentos de Participación Ciudadana y, por lo general, su elaboración ha supuesto un intenso proceso de colaboración entre gobierno municipal y organizaciones ciudadanas ${ }^{23}$.

Ahora bien, originariamente, estos procesos son de carácter estrictamente localista. Surgen y son fomentados por actores políticos y civiles en diversos municipios. Posteriormente, la gran amplitud de su difusión entre otros municipios provocó que la legislación nacional recogiera tales experiencias. En la actualidad, alrededor de tres cuartas partes de los municipios italianos y españoles poseen reglamentos de participación ciudadana ${ }^{24}$.

El desarrollo de marcos generales para la regulación de los procesos de participación ciudadana ha supuesto, a diferencia de las iniciativas desarrolladas en otros países, un proceso simultáneo de democratización e institucionalización a través de oferta de oportunidades de participación. El desarrollo de estrategias parciales como el referéndum o los consejos consultivos, suponen procesos de democratización que abren nuevas posibilidades para que los ciudadanos participen en la toma de de- cisiones, además de las existentes a través de los procesos electorales. En cambio, el desarrollo de Reglamentos de Participación Ciudadana supone un proceso de institucionalización o formalización, por cuanto se generan mecanismos estables de control de la acción política por parte de los ciudadanos. Por tanto, pueden considerarse procesos de constitucionalización a nivel municipal, ya que añaden otros mecanismos de control al que tiene lugar mediante los procesos electorales, ya que tanto el ejecutivo, en este caso el Alcalde o Sindaco, Comisión de Gobierno o Giunta Comunale, como el legislativo, el Pleno Municipal o el Consiglio Comunale, se ven sometidos al control de la ciudadanía ${ }^{25}$.

\section{Comentarios finales}

Los resultados del estudio exploratorio realizado han permitido trazar un esquema básico acerca de las iniciativas locales analizadas, que podrían representarse tal y como aparece en la Ilustración 1. A partir de este esquema podrían plantearse estudios para analizar y evaluar en profundidad la contribución que tales iniciativas locales realizan al aumento de la calidad de las democracias occidentales, tanto en lo que se refiere a la eficacia de la acción gubernamental, como al fomento de la participación ciudadana. Además, en un momento en el que diferentes actores, políticos, civiles o investigadores, ponen de manifiesto la necesidad de rediseñar el lugar y papel de los municipios en los Estados nacionales, y aún más, en la construcción de la Unión Europea, esto puede ser de interés. 


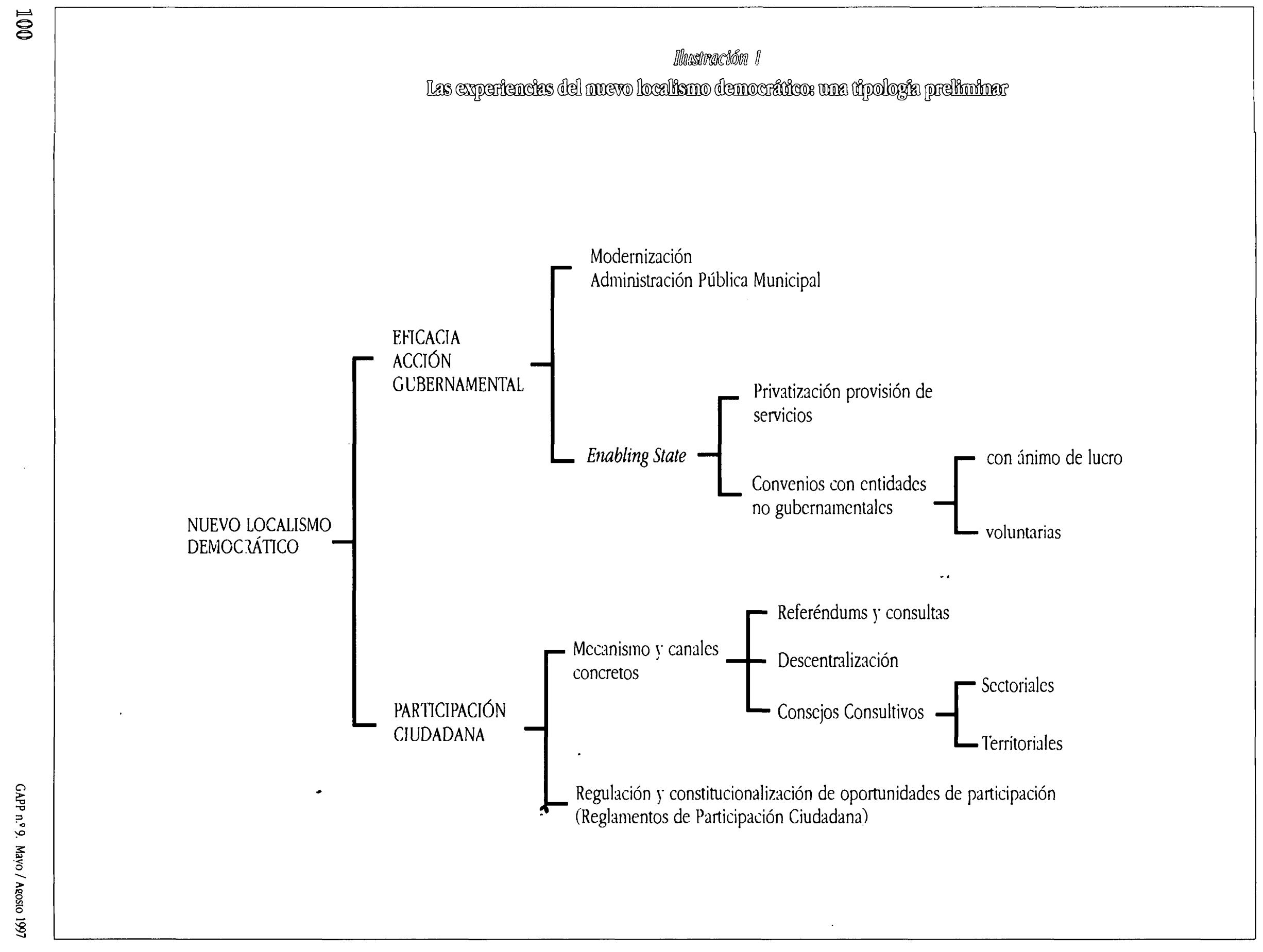


- He de agradecer a Manuel Pérez, Yruela, director del IESA de Andalucía y de la tesis docioral en que se basa parte de este trabajo, sus múltiples sugerencias e indicaciones. Asi como haber realizado una revisión exhaustiva de versiones anteriores del texio.

- Instituto de Estudios Sociales Avanzados de Andalucía/CSIC. Universidad Pablo de Olavide.

'Esta apreciación es generalizada en los autores participacionistas. con independencia de la escuela de pensamiento político en la que se inscriba. Cf., por ejemplo, MaCPHERSON (1991), KFlso (1978) o BarBer (1984). En NAVARRo (1997: 98-102) puede consultarse una breve exposición de este asunto.

? Sobre las competencias de los gobiemos municipales se remite a NoRTOv (1991) y Consejo de Europa (1988).

3 "El estudio exploratorio ha sido realizado mediante diversas técnicas de recogida y análisis de información, tal y como documentación, bibliografia, entrevistas o informantes cualificados. Para una exposición más detallada de este asunto se remite a NavarRo (1997: 45-50).

4 La incidencia de este factor ha sido puesta de manifiesto por STOKER (1991), GRU. NOW (1991) y DENTE (1991).

' Los dos últimos factores han sido destacados por ELCock (1994: 109-114).

${ }^{6}$ En esencia, se trata de reformas encaminadas a mejorar las tareas de comunicación e interacción con los ciudadanos, a través del uso de nuevas tecnologías de la información y comunicación y la reducción de reglas y regulaciones. Con ello se pretende disminuir el tiempo de respuesta a las demandas y, por tanto, aumentar la eficacia en la provisión pública de servicios (GRUNOw, 1991).

' Los principios básicos de este programa son la modemización de la administración municipal y el aumento de su eficacia. Para ello se pastula el planteamiento de objetivos claros y evaluables, la simplificación en los procesos, asi como aumentar el grado de acercamiento y apertura a los ciudadanos, mejorando el acceso a la información, para procurar así ampliar sus posibilidades de elección (Gustafsson, 1991). También pueden consultarse LODDEN (1991) y ROSE (1990).

${ }^{8}$ En HiL. (1994) y, sobre todo, en BlACKMAN (1995), pueden consultarse ejemplos concretos.

'La redacción de Citizen's Chartes se ha extendido desde los gobiemos locales al gobiemo central. Así, en 1991 aparece el White Paper on the Citizen's Chanter (Libro Blanco de las Caras Ciudadanas), redactado por el gobiemo Major. Éste se viene aplicando a politicas sectoriales que desarrolla el gobierno central británico. El principal objetivo que plantea este documento es el desarrollo de programas de reforma que lleven al aumento en la calidad de los servicios, asi como a la incorporación de los ciudadanos en la provisión de éstos. Con ello se pretende aumentar el poder de los ciudadanos como consumidores de servicios. En este senvido, el White Paper senala que a vas Cartas Ciudadanas les concieme dar mayor poder a los ciudadanos. Pero la ciudadanía tiene que ver tanto con nuestras responsabilidades - como padres, por ejemplo, o como vecinos- como con nuestros derechos. La Carta Ciudadana no es una receta para aumentar la acción estatal; es una manifestación de nuestra creencia en el derecho de la gente para estar informados y elegir por ellos mismos. (Primer Minister: The Citizen's Charter: Raising the Standard, Cm. 1599 lHMSO, July 1991], p. 2; citado por PrIOR [1995: 86l). Según David PrIOR el impacto de las Cantas Ciudadanas ha sido menor y diferente de los que se esperaba, como, por ejemplo, la existencia de un desconocimiento generalizado de las Cartas por parte de la población, y el no haber logrado una reducción significativa del gasto público (PRIOR, 1995: 97-100).

Este tipo de procesos suelen estar ligado a una nueva concepción de la ciudadanía procedente de la llamada .Nueva Derecha. Muy brevemente, se trata de una concepción en la que los ciudadanos se definan no por la recepción -pasiva. de servicios, sino por su colaboración en la provisión de éstos, o incluso su autoprovisión. En este sentido se hace referencia a este tipo de mediclas como workfare programs, para diferenciarlos de los welfare programs desartollados tradicionalmente. Sobre las concepciones y discusiones acerca de la ciudadania en la filosofia política contemporánea cf. KYMBLCKA Y NORMAN (1994).

${ }^{10}$ Cf. los trabajos de compilación realizados por BLAIR y STOKER (1991) y CHANDLER (1993).
1: Según un estudio realizado recientemente en la Comunidad Autónoma Andahuza, del total de los servicios prestados por los municipios el $29 \%$ se hace medianie empresas privadas, el $27 \%$ mediante empresas municipales, el $11 \%$ a través de Patronatos y el 33\% restante direcaamente por pare del Ayuntamiento (VAUE CABRERA y ouros, 1991: 76). Según los autores del estudio, existe una tendencia que apunta a que los servicios relacionados con la ubanización y el transporte colectivo de viajeros sean prestados a través de empresas privadas, que parece acentuarse a medida que se incrementa la población de los municipios, siendo patente en el caso de las capitales de provincia. (VAlIE CABRERA y otros, 1991: 87).

En Francia, por ejemplo, la provisión de agua ha sido gestionada por empresas privadas desde finales del siglo XIX, tal y como la Compagnie Générale des Eaux (1853) o la Société Lyonnaise des Eaux (1880). Desde inicios del siglo XX estas empresas se diversificaron y actuaron en ouros sectores, como el de la electricidad (Société Lyonnaise de Eaux et de l'Éclairage). Posteriomente, el crecimiento urbano de los años 50 permitió su extensión, tanto sectorial como teritorialmente (LORRANE, 1991).

12 Según el estudio citado en la nota anterior referido a los municipios andaluces, se tiene que considerando únicamente los servicios de urbanización (limpieza viaria, abastecimiento domiciliario y abastacemiento de mercados), el de transporte colectivo ubano de viajeros y la promoción de viviendas, la prestación se realiza en un $34 \%$ a través de empresas privadas, el $29 \%$ mediante empresas municipales, el $3 \%$ mediante patronatos y el $34 \%$ directamente por pante de los Ayuntamientos (VAlE CABRERA y otros, 1991: 80). Si se comparan con las cifras citadas anteriormente, referidas al conjunto de todos los servicios, se tiene un incremento en los porcentajes de los dos primeros tipos de prestaciones (a través de empresas públicas o privadas), y una disminución en los otros dos (patronatos y Ayuntamientos de forma directa). Esto puede ser indicador de que los servicios de carácter social suelen proveerse a través de patronatos y por parte de los gobiemos municipales directamente, mientras que los relacionados con la planificación urbana tienden a hacerlo mediante empresas, privadas, semi-públicas o públicas.

Según los autores del estudio, el uso de las empresas privadas se centra en los servicios de urbanización, mientras que las empresas públicas se centran en la promoción de viviendas (Valie CaBreRA y otros, 1991: 80-83).

13 Sobre los Estados Unidos cf. Hula (1993) o Chavdure (1993). Según datos del Fiscal Austerity and Urban Innovation Project, el $46 \%$ de las ciudades norteamericanas han hecho del contrato de servicios con empresas privadas una estrategia de austeridad fiscal (Clark, 1996: 27). El caso de Gran Bretaña puede consultarse en Chavdier (1993) o MAXWEL (1993).

14 Algunos ejemplos pueden consultarse en Ramírez (1992) o GruNow (1991).

15 En todo caso, y a diferencia de los casos anteriores, según Bruno DENTE en el caso de Italia los procesos de privatización no adoptan un carácter intencional: el personal contratado temporalmente por la administración pública, una vez termina su relación laboral con ésta, forma cooperativas que más tarde suelen proveer los mismos servicios que cuando formaban parte de la administración municipal, ya sea de una forma autónoma o por encargo de los gobiernos locales. Este autor denomina a este proceso - Estilo Italiano de Privatización. (Privatisation .Italian Style), cf. DevTE (1991).

${ }^{16}$ Cf. Chandler (1993) para el caso de Estados Unidos y GustafFson (1991) para los Países Nórdicos. Según Wolman y Goldsmm, alrededor del $80 \%$ de los municipios norteamericanos pueden convocar referéndums. En el caso de abordarse asuntos de indole económica como, por ejemplo, los impuestos locales, algunos están sujetos a la legislación estatal (Wolmav y GoLSMith, 1992: 128 y ss.).

A nivel nacional se produce la misma dinámica. En Francia e Italia, sobre todo entre los años 70 y 80 , se han usado en algunos casos, mientras que en Alemania y Gran Bretana existe tradicionalmente oposición a su uso. La dinámica política se centra en el Parlamento u órganos municipales del mismo carácter, y no en el uso de fórmulas de paricipación directa tal y como el referéndum (АџUм, 1995: 322-324).

\section{1" Cf. Territoires, $n^{\circ} 358$ para el caso francés. LODDEN (1991) para el caso noruego.}

${ }^{18}$ En este caso puede diferenciarse entre descentralización, cuando se otorga también capacidad de decisión - poder político-, y desconcentración, cuando ello no se produce. Se ha preferido el primer término, ya que se especifican aquellos casos en el que ha existido delegación de competencias. Además, en la literatura que aborda el tema es totalmente mayoritario, por no decir exclusivo, el uso de este término, y no el de desconcentración. 
19 Cf. Grunow (1991) para el caso alemán, Gustafrson (1991) y Jones (1993) para los Países Nórdicos. En Gran Bretaña también se han producido procesos de descentralización. Aunque el objetivo ha sido acercar los servicios a los consumidores más que incorporar a los ciudadanos en los procesos de toma de decisiones (ELCOCK, 1994). Sobre los procesos de descentralización realizados en París y Londres, cf. SAPONARA (1992).

2 Cf. SPENCE (1993) y CENSIS (1988). Actualmente los Consigli di Quartieri quedan regulados por la Ley n. ${ }^{\circ} 142$ de 1990.

$"$ Existen experiencias en otros paises europeos, pero no ha sido una estrategia generalizada. Por ejemplo, la Carta de la democracia local en Marsella (.Charte de la démocratie locale) (PERRIER, 1995).
"En España la Ley Reguladora de Bases de Régimen Local (Ley 7/1985, de 2 de abril), y en Italia la ley sobre el Ordinamento delle autonomie locale (ley n. ${ }^{\circ} 142$, de 8 de julio de 1990).

"Sobre los Reglamentos de Participación Ciudadana en los municipios españoles, cf. NAVARRO (1994). Para el análisis de casos concretos, cf. Femp (1991).

24 Para un análisis detallado del proceso de génesis y desarrollo de este tipo de iniciativas se remite a NAVARRO (1997).

s Sobre la diferencia entre democratización y constitucionalización, cf. ELSTER y SLAGSTAD (1988), especialmente la introducción realizada por ELSTER.

\section{Bibliografia}

AшUm, P. (1995), State and Society in Western Europe, London, Polity Press.

BaRBER, B. (1984), Strong Democracy, California, California University Press.

BATLEY, R. y StOKER, G. (eds.) (1991), Local Government in Europe. Trends and developments, London, MacMillan.

Blackman, T. (1995), Urban Politic in Practice, London, Routledge.

Blar, Ph. (1991), :Trends in local autonomy and democracy, en BATLFY, R. y StokER, G. (eds.), Local Govermment in Europe. Trends and developments, London, MacMillan.

CENSIS (1988), Partecipazione ed efficienza, Milano, Franco Angeli.

Chandler, J.A. (1991), Local Govermment Today, Manchester, Manhester University Press.

ChandLer, J.A. (1993), Local Government in Liberal Democracies. An Introductory Survey, London, Routledge, 1993.

ClaRKE, S. y Galle, G. (1989), Moving towards entrepreneurial state and local economic development strategies: opportunities and barriers, en Policy Studies Joumal, $\mathrm{n}$. 17, pp. 574-598.

CONSEJO DE EUROPA (1988), Allocation of power to the local and regional levels of govermment in the member states of the Council of Europe, Strasbourg, Council of Europe.

DAHL, R. y TuFTE, R. (1973), Size and Democracy, Stanford, Satanford University Press

DENTE, B. (1991), .Italian Local Services: the difficult road towards privatisation, en BATLE, R. y STOKER, G. (eds.), Local Government in Europe. Trends and developments, London, MacMillan, pp. 110-122.

Elcock, H. (1994), Local Government. Policy and Management in Local Authorities, London, Routledge.

ELSTER, J. y SLAGSTAD, R. (eds.) (1988), Constitutionalism and Democracy, Cambridge, Cambridge University Press.

FEMP (1991), Il Jomadas de Participación Ciudadana de la FEMP, Madrid-Granada, FEMP/Ayto. de Granada.

Goetz, E. y Clarke, S. (eds.) (1993), The New Localism. Comparative Urban Politics in a Global Era, London, Sage.

Gustaffson, A. (1991), The Changing Local Government and Politics in Sweden, BATLEY, R. y STOKER, G. (eds.), Local Government in Europe. Trends and developments, London, MacMillan, pp. 170-189.
Grunow, (1991), Costumer-Oriented Service Delivery in German Local Administration, en BATLEY, R. y STOKER, G. (eds.), Local Government in Europe. Trends and developments, London, MacMillan, pp. 73-88.

HiLL, D. (1980), Teoria democrática y régimen local, Madrid, IEAL, 1980.

(1994), Citizens and Cities Urban Policy in the 1990's, London, HarvesterWheatsheaf, 1994.

HuLA, R. (1993), The state reassessed: the privatization of local politics, en GOETz, E. y ClarKE, S. (eds.), The new localism, London, Sage, pp. 22-45.

JONES, B. (1993), Sweden, en CHANDLER, J.A. (ed.), Local Governmentin Liberal Democracies. An Intraductory Survey, London, Routledge.

Kelso, W. (1978), American Democracy Theory, Londres, Greenwood Press.

KInG, S. y PIERRE, J. (eds.) (1990), Challenges to Local Govermment, London, Sage/ECPR.

KYMBUCKA, W. y NORMAN, N. (1994), •Retum of the Citizen: A Survey of Recent Work on Citizenship Theory, en Etbics, 104, pp. 352-381.

LODDEN, P. (1991), -The "Free Local Govemment" Experiment in Norway, en BATLEY, R. y STOKER, G. (eds.), Local Government in Europe. Trends and developments, London, MacMillan, pp. 198-209.

LORRAINE, D. (1991), .Publics goods and private operators in France, en BAmEY, R. y STOKER, G. (eds.), Local Government in Europe. Trends and developments, London, MacMillan, pp. 89-109.

MACPHERSON, (1991), La democracia liberal y su época, Madrid, Alianza Editorial.

MAXWeIL, S. (1993), •El voluntariado escocéso, en MORENo, L. y SaRaSA. S. (eds.), Intercambio social y desarrollo del bienestar, Madrid, CSIC, pp. 197-212.

NavarRo, C. (1994), Encuesta sobre Paricipación Ciudadana de la FEMP. Borrador Informe Base, Documento ed. Trabajo del IESA de Andalucía/CSIC, Córdoba, 1994.

(1997), Innovación Democrática en el Sur de Europa. Las experiencias municipales de Italia y España, Tesis Doctoral (Universidad Complutense de Madrid), Madrid, 1997.

Norron, A. (1991), The Intenuational Handbook of Local and Regional Government. Status, Structure and Resources in Advanced Democracies, Cheltenham, Edward Elgar Publishing Ud, 1991.

PERRIER, (1995), ·Reconstruction démocratique à Marseille, en Territoires, n. 356, pp. 21-24. 
PRIOR, (1995), -Giizen's Charter, en StEWART, J. y STOKER, G. (eds.), Local Govenmment in tbe 1990's, London, MacMillan, pp. 86-103.

Ramfrez, J.L. (1992), la paricipación ciudadana en los paises nórdicos, en Conferencia Europea sobre Participación Ciudadana en los Municipios, Córdoba, 47 de noviembre de 1992.

Rose, R (ed.) (1990), +Nordic Free-Government Experiments: Increased Local Autonomy or Continuated Central Controls, en KIVG, D. y PIERRE, S. (eds.), Cballenges to Local Government, London, Sage/ECPR, pp. 212-241.

SAPONARA, V. (1992), Recenti esperienze di decentramento comunale e sud-comunale nella CEE: le nouve forme di governo delle Aree Meuropoliten de Parigi e Londra, en Confronti, n. 45, pp. 39-45.

SPENCE, R.E. (1993), Italy, en ChnNdifR, J.A. (ed.), Local Govermment in Liberal Democracies. An Introductory Survey, London, Sage.
STEWART, J. y STOKRR, G. (eds.) (1995), Local Gouemment in the 1990's, London, MacMillan.

STOKER, G. (1991), Introduction: Trends in European Local Govemment, en BATEY, $\mathrm{R}$ y STOKER, G. (eds.), Local Goveniment in Europe. Trends and developments, London, MacMillan.

VAUE CABRERA, R. y OTROS (1991), Una apraximación empirica a la realidad municipal de Andalucia, Granada, CEMCI.

VANDEU, L. (1990), Pouwoirs Locaux, Paris, Economica.

Wolman, H. y GolDMisTh, M. (1992), Utban Politios and Policy. A Comparative Approacb, Oxford, Blackwell. 


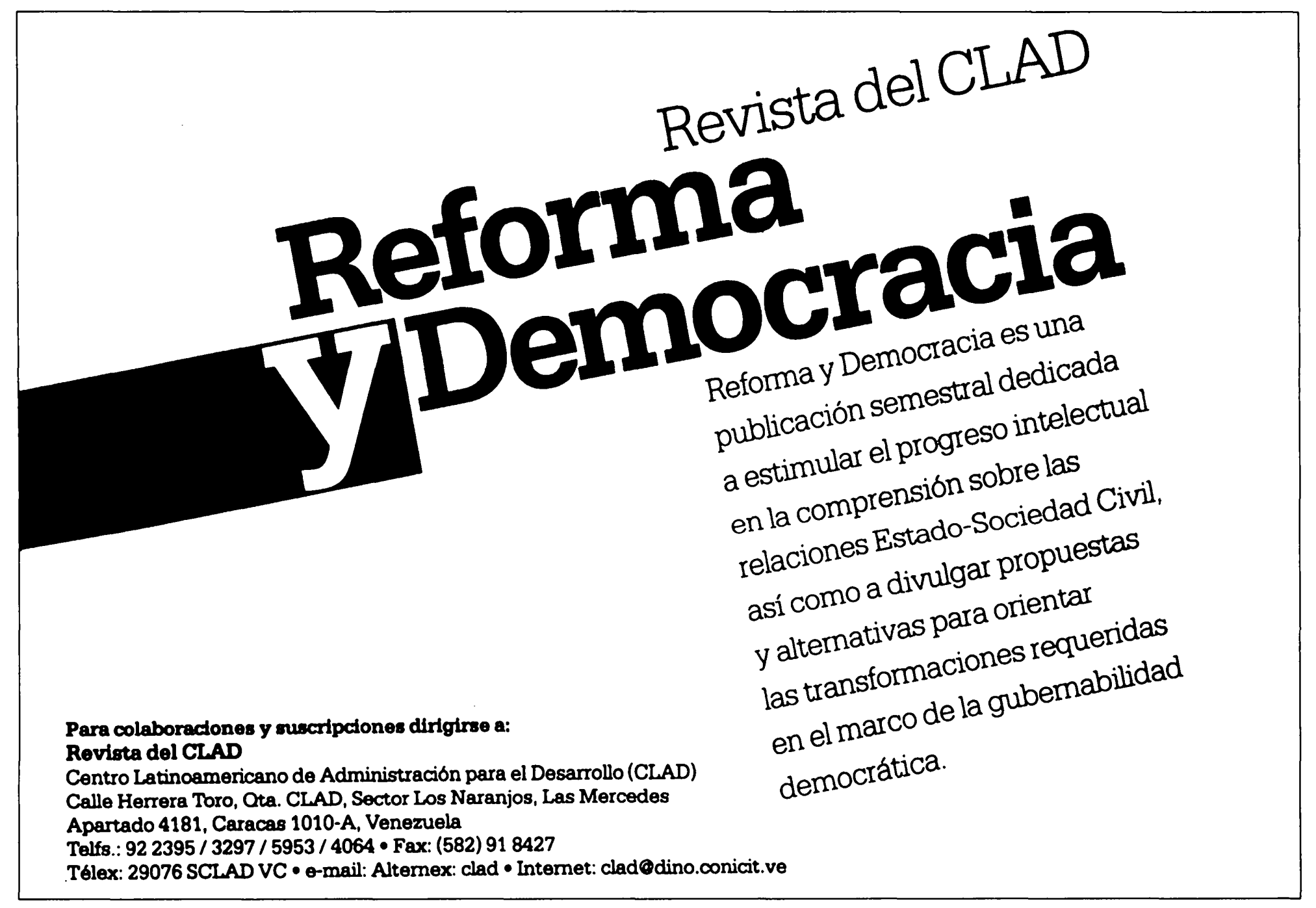

